\title{
Management of Hepatocellular Carcinoma in Japan: Current Trends
}

\author{
Masatoshi Kudo \\ Department of Gastroenterology and Hepatology, Kindai University Faculty of \\ Medicine, Osaka, Japan
}

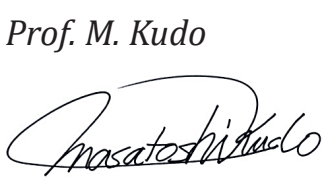

Editor Liver Cancer

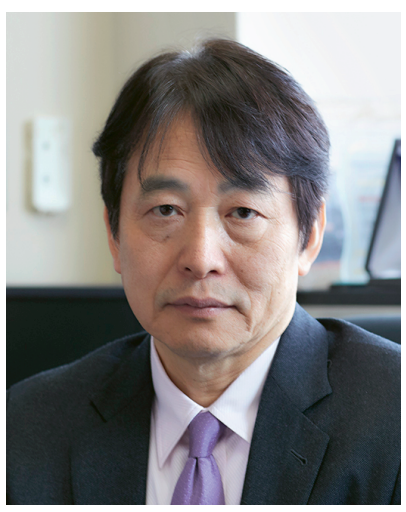

The results of the 20th Nationwide Follow-up Survey of Primary Liver Cancer in Japan were recently published by the Liver Cancer Study Group of Japan [1]. The report included data on 21,075 patients newly registered at 544 institutions across Japan over a 2-year period from January 1, 2008, to December 31, 2009, in addition to 40,769 previously followed patients. Since its establishment in 1965, the Liver Cancer Study Group of Japan has been conducting biennial nationwide liver cancer surveys, with the results of each of the previous 19 surveys published in international journals. The most recent survey, which includes hepatocellular carcinoma (HCC) patients who registered in 2008 and 2009, is no exception, and the basic statistics and results of analysis of follow-up data were reported in Hepatology Research in 2019 [1].

A summary of the findings is as follows. Cases of early-stage HCC have become the majority, with an extremely small number of cases comprising advanced or end-stage liver cancer due to the established nationwide surveillance program in the early 1980s. The initial treatment modality was resection in $37.7 \%$ and ablation in $28.4 \%$ of patients, meaning that curative treatment was performed in as many as $66.1 \%$ of patients. Transarterial chemoembolization was performed as the initial treatment modality in $27.5 \%$ of patients [1]. The proportion of patients who underwent curative treatment is higher than the previously 


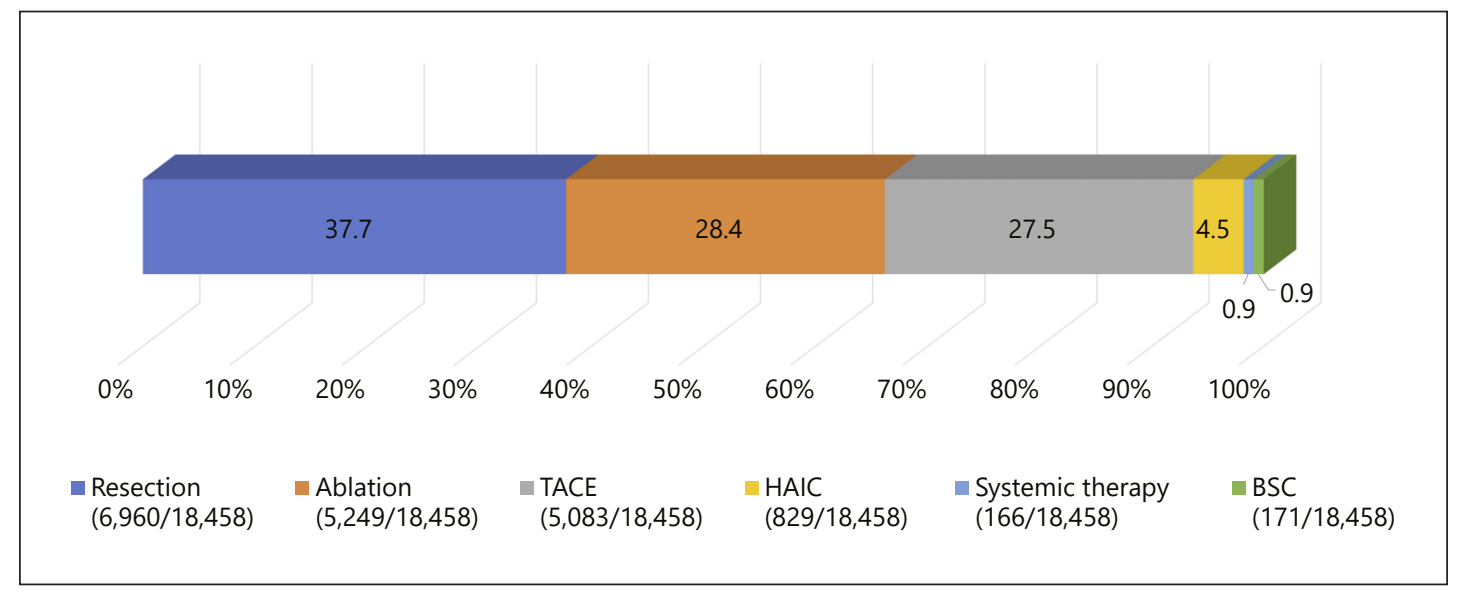

Fig. 1. Initial treatment modality for HCC detected between 2008 and 2009 ( $n=18,458$ ). HCC, hepatocellular carcinoma; TACE, transarterial chemoembolization; HAIC, hepatic arterial infusion chemotherapy; BSC, best supportive care. Modified from Kudo et al. [1].

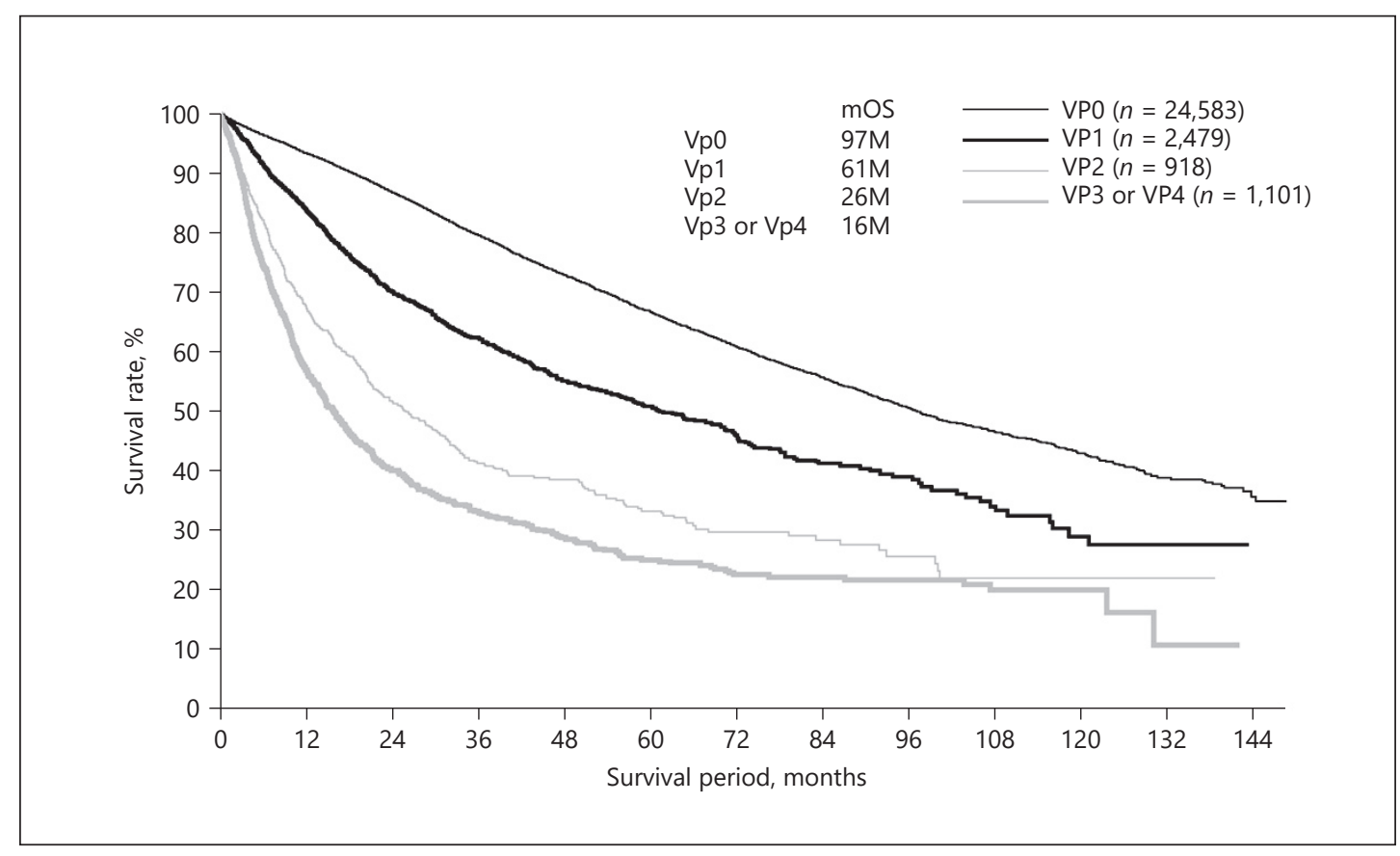

Fig. 2. Overall survival according to grade of portal vein invasion in patients with hepatocellular carcinoma treated with surgical resection $(n=29,081)$. Median OS in patients with Vp0, Vp1, Vp2, and Vp3/Vp4 are 97, 61, 26, and 16 months, respectively; 5- and 10-year survival rates in patients with Vp0, Vp1, Vp2, and Vp3/ Vp4 are 67, 51, 33, and 25, and 43, 29, 22, and 20\%, respectively. mOS, median overall survival; M, months. Modified from Kudo et al. [1].

reported figure [2]. Japan was an early adopter of hepatic arterial infusion chemotherapy (HAIC), and this treatment was performed in $4.5 \%$ of patients with advanced HCC in the 20th survey (Fig. 1) [3, 4]. Because the molecular targeted agent, sorafenib, was approved in Japan in 2009 , the proportion of patients who underwent systemic therapy was still low $(0.9 \%)$. In addition, the proportion of patients who received only best supportive care as the initial treatment was $0.9 \%$, indicating that some form of therapy was offered to many patients in 


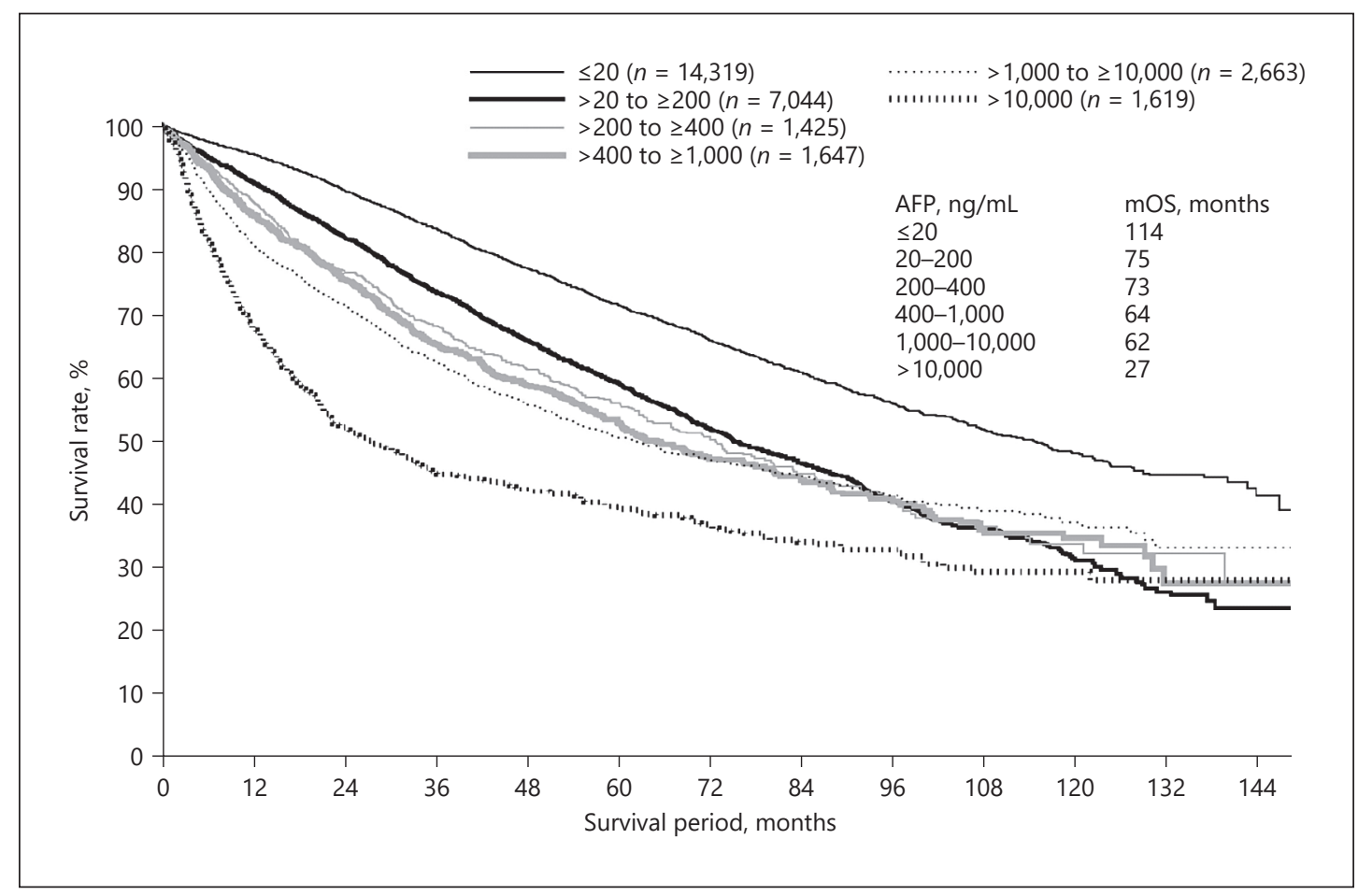

Fig. 3. Overall survival according to serum AFP level in patients with hepatocellular carcinoma treated with surgical resection $(n=28,717)$. mOS, median overall survival. Modified from Kudo et al. [1].

Japan after their initial diagnosis [5]. This was also true for Child-Pugh grade $\mathrm{C}$ and Barcelona Clinic Liver Cancer (BCLC) stage D; the proportion of patients who received best supportive care as the initial treatment after initial detection of HCC was extremely low $(0.9 \%)[5,6]$.

Portal vein tumor thrombosis was revealed to be a factor strongly associated with poor prognosis. When the overall survival (OS) rates of HCC patients who had undergone resection were compared by severity of portal vein invasion, median OS was found to decrease according to the extent of invasion: 97 months in patients with no portal vein invasion (Vp0), 61 months in those with Vp1, 26 months in those with Vp2, and 16 months in those with Vp3-Vp4 (Fig. 2). In addition, AFP level is also a strong predictor of prognosis and can serve as a prognosticator. When the OS of 28,017 HCC patients who had undergone hepatectomy was compared by serum AFP level, median OS decreased as AFP level increased: 114 months in patients with an AFP level $\leq 20 \mathrm{ng} / \mathrm{mL}$, and 27 months in those with an AFP level $>10,000 \mathrm{ng} / \mathrm{mL}$ (Fig. 3). These results clearly show that portal vein invasion and AFP level are extremely important predictors of prognosis as well as prognostic factors in patients with HCC.

Comparison of median OS across four 8-year periods (registration period I, 1978-1985; period II, 1986-1993; period III, 1994-2001; and period IV, 2002-2009) shows clear improvement in median OS over the years: 5 months in period I, 25.6 months in period II, 43.0 months in period III, and 60.8 months in period IV (Fig. 4). Five-year survival rate also steadily improved: $11.9 \%$ in period I, $25.5 \%$ in period II, $39.7 \%$ in period III, and $50.4 \%$ in period IV. These data show a marked improvement in HCC prognosis compared with the results of the previous survey [2]. The above survival results were of patients in Japan with HCC at any stage (from BCLC stage 0 to stage D). It is clear that the complete establishment of a nationwide surveillance program increased the number of patients whose HCC was detected at an early stage; consequently, more HCC patients were able to receive curative therapy. This means that more patients benefited from curative treatment, which resulted in prolonged survival. 


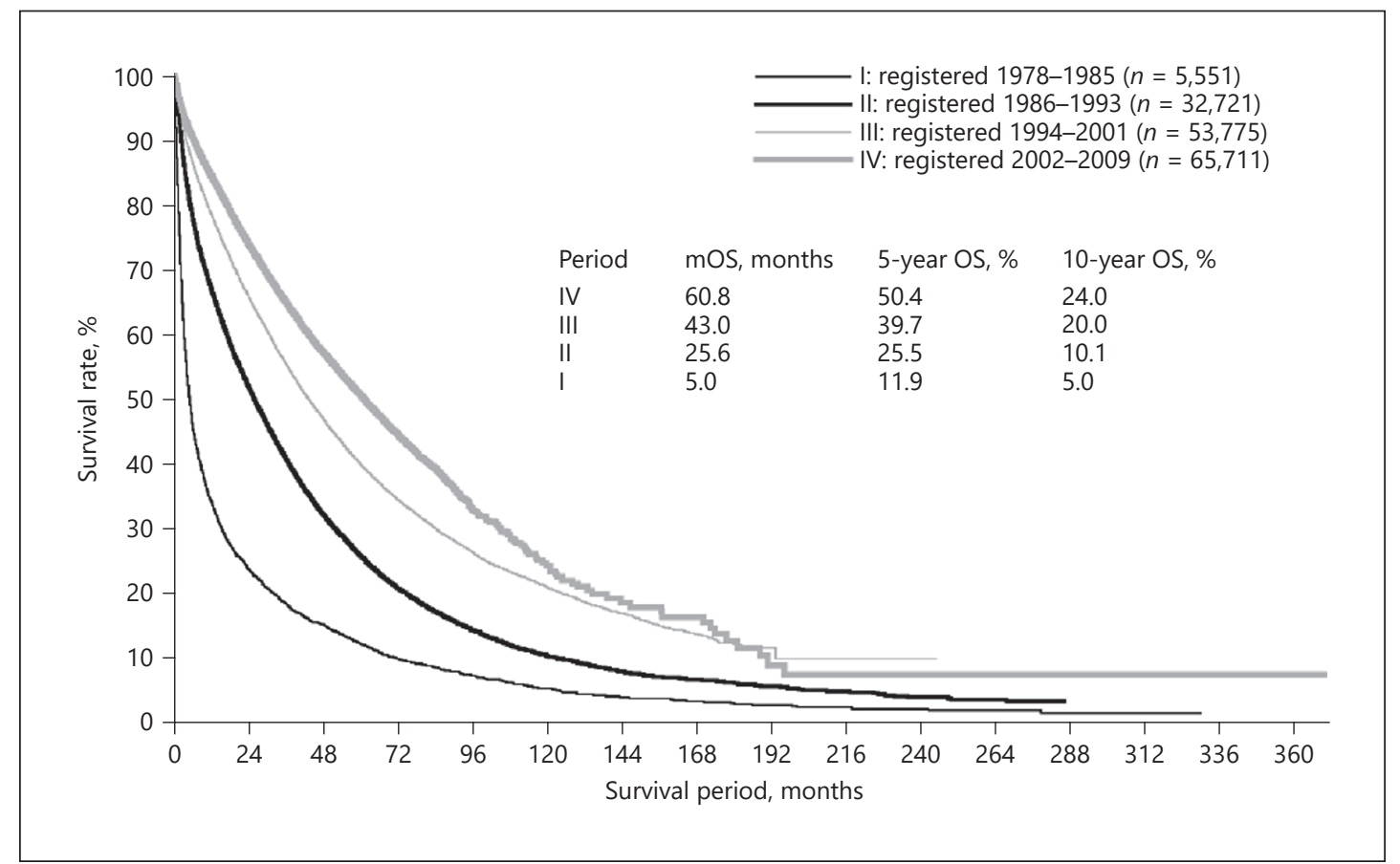

Fig. 4. Improvement of overall survival in all registered patients $(n=157,758)$ between 1978 and 2009 (all patients: BCLC stage 0, A, B, C, and D). Modified from Kudo et al. [1].

Japan leads the world in the outcome of liver cancer treatment, having a considerably higher 5 -year survival rate compared with South Korea (18.9\%) [7], Taiwan (20-22\%), and the USA $(10-15 \%)$ [8-11]. With the approval of sorafenib in 2009 followed by other targeted agents such as lenvatinib, regorafenib, or ramucirumab, a further increase in survival is expected in Japan. The surveillance system and curative treatment approach for HCC in Japan is recognized as a world-leading model [12-14], but HAIC may also have contributed to improvements in prognosis, especially in patients with macrovascular invasion [3]. However, the role of HAIC might be changed in an era of combination immunotherapy, such as atezolizumab plus bevacizumab [15].

\section{Statement of Ethics}

Ethical approval was not applicable.

\section{Disclosure Statement}

M. Kudo has performed lectures for Eisai, Bayer, and MSD. He has received grants from EA Pharma, Eisai, Gilead, Takeda, Otsuka, and Taiho, and has been an advisory consultant for Eisai, Ono, MSD, and BMS.

\section{Funding Sources}

The author has no funding sources to disclose. 
Kudo: Current Trends in Hepatocellular Carcinoma in Japan

\section{References}

1 Kudo M, Izumi N, Kubo S, Kokudo N, Sakamoto M, Shiina S, Tateishi R, Nakashima O, Murakami T, Matsuyama Y, Takahashi A, Miyata H, Takayama T: Report of the 20th Nationwide Follow-up Survey of Primary Liver Cancer in Japan. Hepatol Res. 2019 Oct. DOI: 101111/hepr13438. Epub ahead of print.

2 Kudo M, Izumi N, Sakamoto M, Matsuyama Y, Ichida T, Nakashima O, et al.; Liver Cancer Study Group of Japan. Survival analysis over 28 years of 173,378 patients with hepatocellular carcinoma in Japan. Liver Cancer. 2016 Jul;5(3):190-7.

3 Kudo M, Ueshima K, Yokosuka O, Ogasawara S, Obi S, Izumi N, et al.; SILIUS study group. Sorafenib plus low-dose cisplatin and fluorouracil hepatic arterial infusion chemotherapy versus sorafenib alone in patients with advanced hepatocellular carcinoma (SILIUS): a randomised, open label, phase 3 trial. Lancet Gastroenterol Hepatol. 2018 Jun;3(6):424-32.

4 Kudo M. Treatment of advanced hepatocellular carcinoma with emphasis on hepatic arterial infusion chemotherapy and molecular targeted therapy. Liver Cancer. 2012 Sep;1(2):62-70.

5 Kudo M, Matsui O, Izumi N, Iijima H, Kadoya M, Imai Y, et al.; Liver Cancer Study Group of Japan. JSH Consensusbased clinical practice guidelines for the management of hepatocellular carcinoma: 2014 update by the Liver Cancer Study Group of Japan. Liver Cancer. 2014 Oct;3(3-4):458-68.

6 Kitai S, Kudo M, Nishida N, Izumi N, Sakamoto M, Matsuyama Y, et al.; Liver Cancer Study Group of Japan. Survival benefit of locoregional treatment for hepatocellular carcinoma with advanced liver cirrhosis. Liver Cancer. 2016 Jul;5(3):175-89.

7 Park JW; Korean Liver Cancer Study Group and National Cancer Center. [Practice guideline for diagnosis and treatment of hepatocellular carcinoma]. Korean J Hepatol. 2004 Jun;10(2):88-98. Korean.

8 Altekruse SF, McGlynn KA, Dickie LA, Kleiner DE. Hepatocellular carcinoma confirmation, treatment, and survival in surveillance, epidemiology, and end results registries, 1992-2008. Hepatology. 2012 Feb;55(2): 476-82.

9 Ries LA, Melbert D, Krapcho M, et al., editors. SEER Cancer Statistics Review, 1975-2005 [cited 2012 Feb 27]. Bethesda (MD): National Cancer Institute; 2008. Available from: seer.cancer.gov/csr/1975_2005/.

10 American Cancer Society. Cancer facts and figures 2012. Available from: https://www.cancer.org/research/ cancer-facts-statistics/all-cancer-facts-figures/cancer-facts-figures-2012.html

11 El-Serag HB. Hepatocellular carcinoma. N Engl J Med. 2011 Sep;365(12):1118-27.

12 Kudo M. Japan's successful model of nationwide hepatocellular carcinoma surveillance highlighting the urgent need for global surveillance. Liver Cancer. 2012 Nov;1(3-4):141-3.

13 Kudo M. Management of hepatocellular carcinoma in Japan as a world-leading model. Liver Cancer. 2018 May; $7(2): 134-47$.

14 Kudo M. Surveillance, diagnosis, treatment, and outcome of liver cancer in Japan. Liver Cancer. 2015 Mar; 4(1): 39-50.

15 Cheng AL, Qin S, Ikeda M, Galle PR, Ducreux M, Zhu AX, et al. IMbrave150: efficacy and safety results from a ph III study evaluating atezolizumab (atezo) + bevacizumab (bev) vs sorafenib (Sor) as first treatment (tx) for patients (pts) with unresectable hepatocellular carcinoma (HCC). Ann Oncol. 2019;30 suppl 9:183-202. 